\title{
Regulation of autophagy during mitosis
}

\author{
Yikang Gong ${ }^{1}$ * \\ ${ }^{1}$ College of Nature Science, University of Massachusetts Amherst, Amherst Massachusetts 01003, U.S.
}

\begin{abstract}
Based on observations of mitosis process, it has been implied that autophagy is repressed. Because the integrity of chromosomes and proteins are significant for cell cycle, the repression of autophagy serves as a protection. However, the mechanistic detail of whether and how autophagy is regulated during mitosis remains controversial. In this review, we aim to analyse and review the recent literature on the regulation of autophagy during mitosis. According to researches, autophagy is repressed via inhibitory phosphorylation of autophagy genes by CDK1, CUL4-RING ubiquitin ligases-mediated and WIPI2 polyubiquitination and proteasomal degradation. In addition, the recent evidence that indicates autophagy is repressed will be reviewed.
\end{abstract}

\section{Introduction}

Autophagy is a "self-eating" process of the cell that is generally induced by various stress conditions. Autophagy is a conserved function in which cytoplasmic contents are engulfed by a double-membrane structure named autophagosome and then fuses with lysosome for degradation [1-2]. In recent years, there is emerging evidence demonstrating that the WIPI (WD-repeat protein domain, phosphoinositide interacting) family plays an important role in facilitating the nucleation and expansion of phagophore membranes [3-4]. It has been accepted that the autophagic process is regulated by a group of proteins coded by the autophagyrelated (ATG) genes [5-6]. There are convincing evidences that WIPI2 (WD repeat domain, phosphoinositide interacting 2) mediates the recruitment of the ATG12-ATG5ATG16L1 complex to the class III phosphatidylinositol 3-kinase-positive omegasome by directly interacting with ATG16L1, and such interaction is indispensable for LC3 lipidation and autophagosome biogenesis in starvation induced autophagy. Up to date, the relationship between autophagy and cell cycle remains ambiguous. It remains unclear or controversial whether and how cell cycle regulates autophagy. It has been reported that autophagy is activated during mitosis [7-8]. In contrast, there are compelling evidence showing impaired autophagy during mitosis. In this review, the function of WIPI2 polyubiquitination and CDK1 CUL4RING ubiquitin ligases-mediated is discussed.

\subsection{The three types of autophagy}

Autophagy has three different types: macroautophagy, the autophagy that is generally referred to, microautophagy, and chaperone-mediated autophagy [9]. Autophagy defects have been linked to many diseases including, cancer, Alzheimer's, Huntington's, and many others [10].

Microautophagy is a process of certain cytosolic cargoes directly taken up by lysosomes in mammalian cells or vacuole in Yeast via invagination of the lysosome [11]. This type of autophagy thus does not require the formation of double-membrane autophagosomes. The exact mechanism of microautophagy is not well characterized.

Chaperone-mediated autophagy is a selective degradation process with the help of cytosolic chaperones. In chaperone-mediated autophagy, hsc70 recognizes the target sequence KFERQ in cytosolic proteins and targets them to the lysosome for internalization. Once the cargoes bind to lysosome receptor Lamp2A, they will be unfolded and translocated into the lumen of the lysosome for recycling [12].

Lastly, macroautophagy is different from the above two types of autophagy so that macroautophagy encapsulates cargoes in double-membrane vesicles called autophagosomes and degrades the cargoes via the fusion of the autophagosome with the lysosome, release of the autophagosome contents [9]. Macroautophagy is the most intensively studied autophagy, and the term "autophagy" generally refers to "macroautophagy". There are more than 40 autophagy genes (Atg) that regulate the macroautophagy process. Different types of autophagy genes are summarized below with functions in Table 1. Autophagy genes are listed with functional groups. Autophagy genes are classified into 5 major groups with different functions listed below. One major function of each group in autophagy is provided.

Table 1. Different types of autophagy genes and their functions

\begin{tabular}{|c|c|c|}
\hline Name & $\begin{array}{c}\text { Autophagy } \\
\text { genes }\end{array}$ & Function \\
\hline Atg1(ULK1) & ULK1, & Phosphorylation of \\
kinase complex & FIP200, & downstream pathway \\
\hline
\end{tabular}

\footnotetext{
${ }^{*}$ Corresponding author: yikanggong@umass.edu
} 


\begin{tabular}{|c|c|c|}
\hline & ATG13, & factors \\
& Atg17, & \\
& Atg29, & \\
& Atg31, & \\
& ATG101 & \\
& Beclin 1, & \\
& VPS34, & \\
& VPS15, & \\
ATG14, & Nucleation for \\
PI3K complex & NRBF2, & phagophore formation \\
& UVRAG & \\
& Atg9, & \\
& ATG2, & \\
WIPI4 & \\
\hline Atg9 cycling & Atg9, & Providing with \\
system & ATG2, & membrane sources for \\
& WIPI4 & phagophore expansion \\
\hline & Atg12, & \\
Atg8-Atg12 UB & Atg8, Atg5, & \\
conjugation & Atg7, & Autophagosome \\
systems & Atg16L, & elongation and \\
& Atg4, & completion \\
& Atg3 & \\
\hline Autophagosome- & RAB & \\
Gysosome fusion & GTPases, & Autophagosome and \\
complex & HOPS, & lysosome fusion \\
SNARES & \\
\hline
\end{tabular}

\section{Autophagy in mitosis}

Autophagy is a cellular catabolic process in which lysosomes degrade cellular contents according to various stress conditions, including nutrient starvation, protein aggregation, viral infection, and others [12]. In addition to maintaining cell homeostasis, autophagy has also been reported to participate in regulating cell cycle progression under nutritional deficiency [10].

Mitosis is when a eukaryotic cell divides the chromosomes in its nucleus evenly into two daughter cells. The mitotic phase is where nuclear is divided, and new cell structures are distributed to daughter cells [13]. The cell cycle regulation is essential for eukaryotic life to faithfully pass genetic information from one generation to the next. It is been hypothesized that autophagy is inhibited during mitosis [14]. The obvious logical reasoning for this hypothesis is that autophagy is largely non-selective; during mitosis in mammals, the nuclear envelope is broken, which creates the risk that the DNA could be degraded by autophagy not be inhibited. Therefore, it is been hypothesized that autophagy is inhibited during mitosis. Here, we will summarize recent advances that support or contradicts this hypothesis.

\subsection{Inhibitory phosphorylation of autophagy genes by CDK1 during mitosis}

mTORC1 is the master regulator of autophagy [11]. During interphase and nutrient-rich conditions, mTORC1 inhibits autophagy flux by inhibitory phosphorylation of ULK1, Atg13, and TFEB, inhibiting autophagy initiation and lysosome biogenesis. During energy or nutrient deprivation conditions, mTORC1 is inhibited, and autophagy is activated. During mitosis, mTORC1 is also inhibited, but autophagy is inactive, which indicates that there is another regulator of autophagy that overrides the master role of mTORC1 in autophagy during mitosis [15].

Odle et al. showed that mitotic phosphorylation of autophagy genes and regulators, including ULK1, ATG13, ATG14, and TFEB, depends on CDK1 but independent of $\mathrm{mTORC} 1$, and $\mathrm{CDK} 1$ is the master regulator of autophagy during mitosis over mTORC1 [15]. Therefore, autophagy suppression still happens even during nutrient deprivation.

Interphase or stress conditions

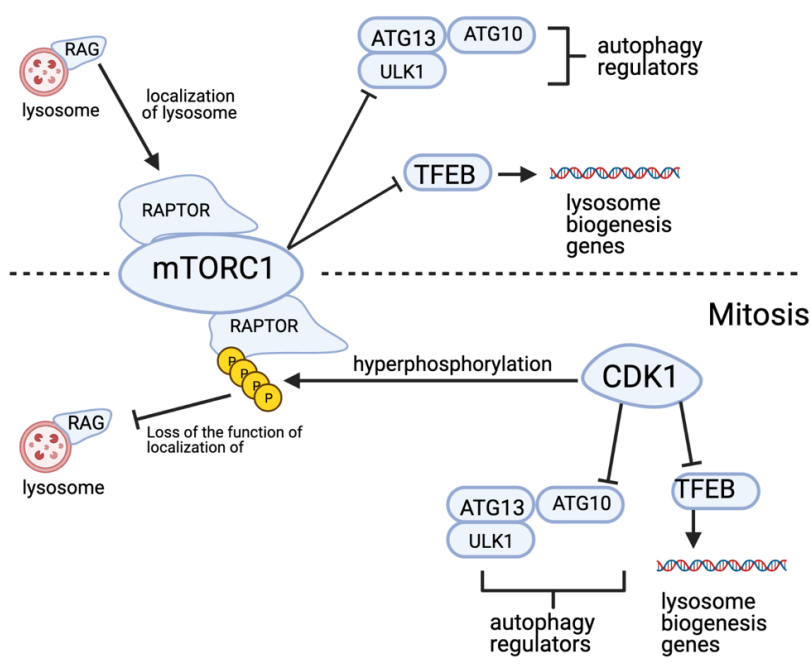

Fig. 1. Regulation of autophagy by CDK1 during mitosis.

As shown in Fig. 1. this schematic model describes CDK1 overrides the function of mTORC1 during mitosis and leads to inhibition of autophagy flux by inhibition of TFEB translocation and inhibitory phosphorylation of ULK1, Atg13, and Atg10.

How is mTORC1 inhibited during mitosis? It turns out during mitosis, RAPTOR, which is essential for mTORC1 localization to the lysosome and its activation, is hyperphosphorylated. Phosphorylation of RAPTOR by CDK1 inhibits its function in the regulation of mTORC1 localization to the lysosome. Therefore, during mitosis, mTORC1 is failed to localize to the lysosome, and its kinase activity is inhibited [15].

Early autophagy markers Atg13 and WIPI2 are observed using immunofluorescence microscopy. The authors identified that Atg13 and WIPI2 puncta numbers are reduced during mitosis compared to interphase or unsynchronized cells, which indicates that autophagy is inhibited. Using immunofluorescence staining with different markers, the authors showed that during mitosis, mTORC1 is not colocalized with lysosome marker Lamp2 while it is during interphase. By observing band shift in western blot for ULK1, Atg13, Atg14, and TFEB in different conditions, the authors assess the activity of mTORC1 and CDK1. By showing the band shift in these autophagy genes even when mTORC1 is inhibited, the authors opened the door for identifying a new kinase for 
this inhibitory phosphorylation [15]. For TFEB activity, the nuclear/cytosolic translocation is assessed during different conditions. Using CDK1 specific inhibitors, in vitro kinase assay, and using synchronized cells, it is confirmed that CDK1 phosphorylates ULK1, Atg13, Atg14, and TFEB inhibit autophagy during mitosis.

In addition to this, there is another recent report showed that CDK $1 / 5$ inhibits autophagy by inhibitory phosphorylation of $\mathrm{Vps} 34$, an essential subunit of class III PtdIns3 kinase [16]. Vps34 phosphorylation on Thr159 by CDK 1 abolishes its interaction with Beclin 1 to reduce the production of PtdIns3P, which is important for autophagy flux. In addition to autophagy, this phosphorylation of Vps34 during mitosis may also regulate other intracellular protein trafficking and glycogen synthesis pathways.

\subsection{WIPI2 ubiquitination and degradation during mitosis}

Autophagy is also suppressed during mitosis via CUL4RING ubiquitin ligases-mediated WIPI2 polyubiquitination and proteasomal degradation [14]. Autophagy is believed to be repressed via the activation of CRL4s during mitosis. Such suppression is caused by ubiquitination and proteasomal degradation of WIPI2, which the author suggests is regulated by CRL4s. The authors found out that during mitosis, the number of GFP-LC3 puncta is less when compared to the interphase and cytokinesis as the GFP-LC3 can serve as a lysosome marker an indispensable element of autophagy. Such phenomena of less GFP-LC3 puncta counts might indicate the level of autophagy is low during mitosis [14].

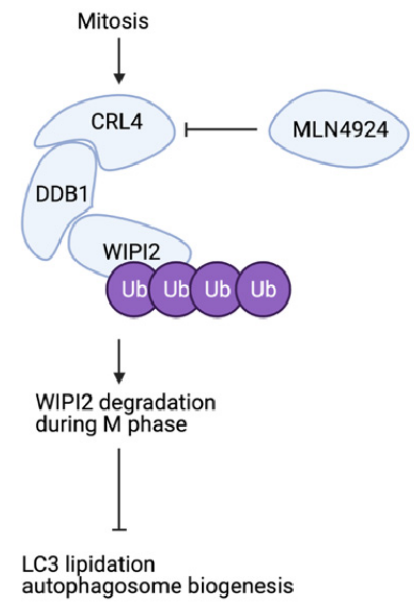

Fig. 2. Inhibition of Autophagy via WIPI2 polyubiquitination and degradation during mitosis.

As shown in Fig. 2. during mitosis, E3 ubiquitin ligase CRL4 recruits WIPI2 with the help of DDB1, polyubiquitinates it, which results in the degradation of WIPI2. Without WIPI2 during mitosis, Autophagy is inhibited.

The authors further studied the phenomenon using biochemical assays. A reduction of autophagy flux is detected via the analysis LC3-I/LC3-II ratio in cells with nocodazole (NOC), a microtubules polymerization inhibitor that arrests cells in the M phase. What is the mechanism of this reduction of autophagy flux during mitosis? Surprisingly, the authors discovered that the essential autophagy gene WIPI2 is downregulated during mitosis via polyubiquitination mediated degradation.

During mitosis, CUL4-RING ubiquitin ligases (CRL4s) are activated via neddylation. Considering previous reports of E3 ligase-mediated degradation of autophagy-related genes and lower WIPI2 levels during mitosis, the authors tested the possibility of WIPI2 ubiquitination by CRL4s. The authors discovered that WIPI2 interacts with CRL4 in vivo and in vitro, which is required for its ubiquitination. It is also showed that CRL4 regulates WIPI2 ubiquitination both in vitro and in vivo, which further confirms the direct effect. Plus, the knockdown of CRL4s led to the rescue of WIPI2, which further confirms that CRL4s ubiquitinate it.

What happens if one forcefully activates autophagy during mitosis? To test this idea, the authors treated the cell with NOC only or NOC+ MLN4924, an inhibitor of CRL4s. MLN4924 dramatically reduced the percentage of mitosis cells, which indicates that forceful activation of autophagy may lead to mitosis slippage. Further experiments showed that it causes sentences and cell death.

\section{Conclusion and future perspectives}

Autophagy has gained tremendous interest in recent years, especially after the Nobel Prize in Physiology and Medicine of 2016 is awarded to Yoshinori Ohsumi for his discoveries in the mechanism of autophagy. Recent advances in our understanding of autophagy, especially its role in cancer and Alzheimer's disease, raised the interest that if autophagy can be targeted to treat these diseases.

In the past years, tremendous effort has been given to understanding the role of autophagy in cancer. As summarized in this review article, autophagy is inhibited during mitosis, which can be a hint and important information in targeting autophagy in cancer. However, it is still too early to dictate if autophagy can be targeted to treat cancer for multiple reasons. First, autophagy is seen as a double-edged sword in cancer because it is activated in some cancer types while inhibited in others. Second, many autophagy genes play different roles during cancer other than regulating autophagy, making it hard to target autophagy genes for cancer therapy due to potential strong side effects. Finally, so far, no good assays for monitoring autophagy in organ, not to mention whole-body level, exist, making it challenging to monitor drug effect.

This paper mainly reviews the novel research suggests a system-wide repression of autopagosomes synthesis during mitosis. The mechanism of autophagy regulation during mitosis is discovered recently. Autophagy is generally inhibited during mitosis by two sets of mechanisms: First, inhibitory phosphorylation of Atg genes such as ULK1 and inhibition of lysosome biogenesis regulator TFEB by CDK1. Second, 
polyubiquitination and degradation of autophagy gene WIPI2 during mitosis leads to inhibition of autophagosome biogenesis. It is possible that other mechanisms of autophagy regulation during mitosis, especially in certain cell types, exist and need further indepth research. Another remaining question in autophagy regulation during mitosis is: Are selective and non-selective autophagy pathways both inhibited during mitosis?

\section{References}

1. N. Mizushima, \& M. Komatsu, Autophagy: renovation of cells and tissues. Cell, 147(4), 728741 (2011)

2. H. M. Shen, \& N. Mizushima, At the end of the autophagic road: an emerging understanding of lysosomal functions in autophagy. Trends Biochem Sci, 39(2), 61-71 (2014)

3. H. E. Polson, J. de Lartigue, D. J. Rigden, M. Reedijk, S. Urbé, M. J. Clague, \& S. A. Tooze, Mammalian Atg18 (WIPI2) localizes to omegasome-anchored phagophores and positively regulates LC3 lipidation. Autophagy, 6(4), 506-522 (2010)

4. C. A. Lamb, T. Yoshimori, \& S. A. Tooze, The autophagosome: origins unknown, biogenesis complex. Nature reviews. Mol. Cell Biol. 14(12), 759-774 (2013)

5. Y. Ohsumi, \& N. Mizushima, Two ubiquitin-like conjugation systems essential for autophagy. Semin. Cell Dev. Biol., 15(2), 231-236 (2004)

6. Y. Chen, J. He, M. Tian, S. Y. Zhang, M. R. Guo, R. Kasimu, J. H. Wang, \& L. Ouyang, UNC51-like kinase 1, autophagic regulator and cancer therapeutic target. Cell Prolif., 47(6), 494-505 (2014)

7. L. Liu, R. Xie, S. Nguyen, M. Ye, \& W. L. McKeehan, Robust autophagy/mitophagy persists during mitosis. Cell cycle, 8(10), 1616-1620 (2009)

8. Z. Li, X. Ji, D. Wang, J. Liu, \& X. Zhang, Autophagic flux is highly active in early mitosis and differentially regulated throughout the cell cycle. Oncotarget, 7(26), 39705-39718 (2016)

9. X. Wen, \& D. J. Klionsky, An overview of macroautophagy in yeast. J Mol Biol, 428, 16811699 (2016)

10. Y. Yang, \& D. J. Klionsky, Autophagy and disease: unanswered questions. Cell Death Differ., 27(3), 858-871 (2020)

11. W. Cao, J. Li, K. Yang, \& D. Cao, An overview of autophagy: Mechanism, regulation and research progress. Bull Cancer (2021)

12. N. Mizushima, B. Levine, A. M. Cuervo, \& D. J. Klionsky, Autophagy fights disease through cellular self-digestion. Nature, 451(7182), 1069-1075(2008)

13. K. Collins, T. Jacks, \& N. P. Pavletich, The cell cycle and cancer. Proc Natl Acad Sci U S A, 94(7), 2776-2778 (1997)
14. G. Lu, J. Yi, A. Gubas, Y. T. Wang, Y. Wu, Y. Ren, M. Wu, Y. Shi, C. Ouyang, H. W. S. Tan, T. Wang, L. Wang, N. D. Yang, S. Deng, D. Xia, R. H. Chen, S. A. Tooze, \& H. M. Shen, Suppression of autophagy during mitosis via CUL4-RING ubiquitin ligases-mediated WIPI2 polyubiquitination and proteasomal degradation. Autophagy, 15(11), $1917-$ 1934 (2019)

15. R. I. Odle, S. A. Walker, D. Oxley, A. M. Kidger, K. Balmanno, R. Gilley, H. Okkenhaug, O. Florey, N. T. Ktistakis, \& S. J. Cook, An mTORC1-to-CDK1 Switch Maintains Autophagy Suppression during Mitosis. Mol. Cell., 77(2) (2020)

16. T. Furuya, M. Kim, M. Lipinski, J. Li, D. Kim, T. Lu, Y. Shen, L. Rameh, B. Yankner, L. H. Tsai, \& J. Yuan, Negative regulation of $\mathrm{Vps} 34$ by $\mathrm{Cdk}$ mediated phosphorylation. Mol. Cell., 38(4), 500511 (2010) 